\section{IN BRIEF}

\section{$\Rightarrow$ IMMUNOTHERAPY}

\section{Faecal transplantation reverses colitis}

Observations from two patients receiving ipilimumab monotherapy and nivolumab plus ipilimumab provide evidence of the feasibility of faecal microbiota transplantation (FMT) for the resolution of immune-checkpoint inhibitor-induced colitis. Both patients had grade $\geq 2$ colitis of a noninfectious aetiology. Stool samples were obtained from a single healthy donor with no gastrointestinal symptoms, sterile filtered, dissolved in a saline solution and applied directly to the caecum of each patient via colonoscopy. The first patient to receive FMT had complete resolution of colitis after a single dose, while the second patient had an initial partial improvement, albeit with ulceration and abdominal pain, which improved to complete resolution following a second FMT. 16s RNA sequencing data confirmed that bacteria initially present in the donor's, but not the host's, intestinal microbiota were incorporated into the host's microbiota after transplantation. These findings warrant further investigation in a larger cohort of patients.

ORIGINAL ARTICLE Wang, Y. et al. Fecal microbiota transplantation for refractory immune checkpoint inhibitor-associated colitis. Nat. Med. https://doi.org/10.1038/ s41591-018-0238-9 (2018).

\section{$\Rightarrow$ HAEMATOLOGICAL CANCER}

\section{Donors with CHIP are safe for allogeneic HSCT}

Data from a study involving 500 related allogeneic haematopoietic stem cell transplantation (HSCT) donors demonstrate that the presence of clonal haematopoiesis of indeterminate potential (CHIP) mutations has no effect on overall survival of the recipients. A total of $92 \mathrm{CHIP}$ mutations were identified in 80 donors (16\%), with a median variant-allele frequency of $5.9 \%$. The majority of patients (70) had a single detectable mutation. Patients who received stem cells harbouring CHIP mutations had an increased risk of graft-versus-host disease (HR 1.73, 95\% Cl 1.21-2.49; $P=0.003)$ and a reduced cumulative incidence of relapse and disease progression (multivariate $\mathrm{HR} \mathrm{0.63,95 \%} \mathrm{Cl} 0.41-0.98 ; P=0.042$ ). These findings suggest that the presence of one or more CHIP mutations should not preclude stem cell donation.

ORIGINAL ARTICLE Frick, M. et al. Role of donor clonal hematopoiesis in allogeneic hematopoietic stem-cell transplantation.J. Clin. Oncol. https://doi.org/10.1200/ JCO.2018.79.2184 (2018)

\section{$\Rightarrow$ LUNG CANCER}

\section{Lorlatinib effective in multiple settings}

Data from a phase II study investigating the efficacy of the ALK and ROS1 tyrosine-kinase inhibitor (TKI) lorlatinib reveal encouraging response rates in patients with $A L K$-rearranged and/or ROS1-rearranged non-small-cell lung cancer (NSCLC) in multiple settings. The majority ( $90 \%$ ) of patients with treatment-naive disease had a response to lorlatinib. Objective responses were observed in $69.5 \%$ of those who previously received crizotinib and in $32.1-47 \%$ of patients who received at least one previous other ALK-TKI with or without chemotherapy. Similar intracranial response rates were observed in patients with brain metastases, thus confirming the brain penetrance of this agent. Hyperchlolesterolaemia and hypertryglyceridaemia were the most common grade $\geq 3$ adverse events (both in $16 \%$ of patients). These data suggest that lorlatinib could be effective in both the first-line and later-line treatment settings. Extended follow-up data from this trial are eagerly awaited.

ORIGINAL ARTICLE Solomon, B. J. et al. Lorlatinib in patients with ALK-positive non-small-cell lung cancer: results from a global phase 2 study. Lancet https:// doi.org/10.1016/S1470-2045(18)30649-1 (2018)

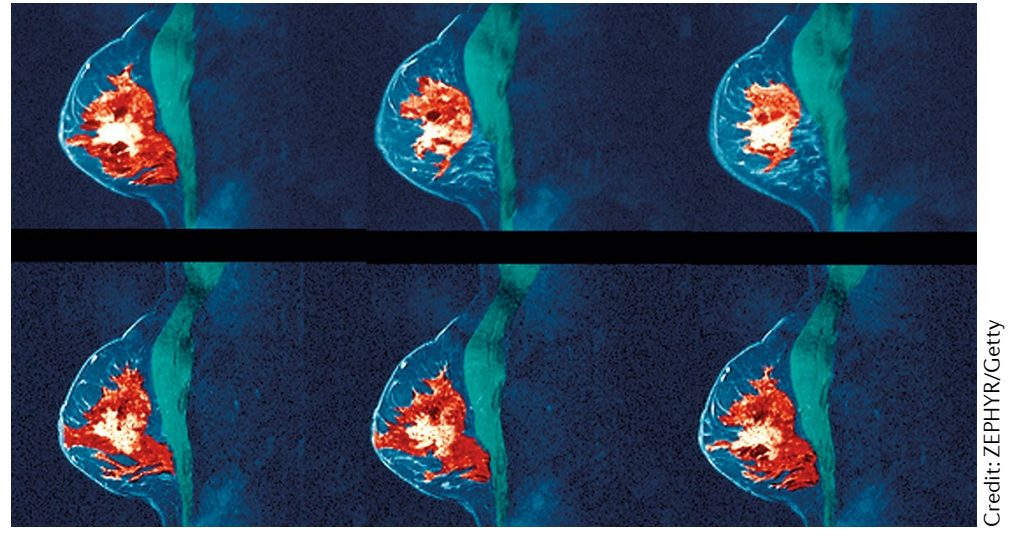

BREAST CANCER

\title{
Setting dictates efficacy of pembrolizumab in TNBC
}

Despite revolutionizing the management of various solid tumours, more limited success has thus far been achieved with immune-checkpoint inhibition in women with advanced-stage breast cancer. Now, data from two cohorts of KEYNOTE-086, a phase II trial designed to explore the efficacy of monotherapy with the antiprogrammed cell death protein 1 (PD-1) antibody pembrolizumab in different treatment settings in women with triple-negative breast cancer (TNBC), provide further insight on the optimal use of these agents.

Women in cohort A (with previously treated metastatic TNBC, $n=170$ ) and in cohort B (with previously untreated, programmed cell death 1 ligand 1 (PD-L1)-positive metastatic TNBC, $n=84$ ) received intravenous pembrolizumab on a 3-weekly basis for up to 2 years. The primary end point in cohort A was safety and in cohort B was safety and overall response rate.

Treatment-related adverse events of any grade were reported in $60.6 \%$ and $63.1 \%$ of women in cohort $A$ and cohort B, respectively, with $12.9 \%$ and $9.5 \%$ having grade $\geq 3$ adverse events. Notably, overall response rates (ORRs) were $5.3 \%$ in cohort A $(5.7 \%$ in women with PD-L1-positive disease), compared with $21.4 \%$ in cohort B and nearly half (44.4\%) of these responses were ongoing at the time of analysis. Median overall survival durations were 9.0 months and 18.0 months in cohort A and cohort B, respectively.
Lead author Sylvia Adams highlights: "The most striking observation is that clinical activity is vastly different by line of therapy." This observation has important implications, both for patient management and for future research. Even in the first-line setting, ORRs to pembrolizumab were lower than those typically associated with chemotherapy; however, among women who did respond, many of the responses were more durable and ongoing at the time of analysis. This finding highlights the need for robust biomarkers of responsiveness to pembrolizumab. Furthermore, the ORRs observed in this trial might be improved upon by the addition of pembrolizumab to chemotherapy.

Adams summarizes: "This is the largest study of single-agent PD-1PD-L1 inhibition in metastatic breast cancer to date," adding "while response rates are lower than those observed with chemotherapy, the durability of responses and associated survival is impressive and confirms that immunotherapy is a game changer (or at least a new treatment option) for women with TNBC and can improve survival in at least a subset of patients." Peter Sidaway

ORIGINAL ARTICLES Adams, S. et al. Pembrolizumab monotherapy for previously treated metastatic triple-negative breast cancer: cohort A of the phase 2 KEYNOTE-086 study. Ann. Oncol. https://doi.org/ 10.1093/annonc/mdy517 (2018) | Adams, S. et al. Pembrolizumab monotherapy for previously untreated, PD-L1-positive, metastatic triplenegative breast cancer: cohort $B$ of the phase 2 KEYNOTE-086 study. Ann. Oncol. https://doi.org/ 10.1093/annonc/mdy518 (2018) 